\title{
Bronquiolitis obliterante con neumonía organizativa idiopática. Utilidad y rentabilidad de técnicas y pro- cedimientos diagnósticos en una serie de 20 pacientes
}

\author{
J. JAREÑO ESTEBAN, E. ZAMORA GARCÍA*, Mª J. CHILLÓN MARTÍN, \\ E. PÉREZ AMOR*, F. VILLEGAS FERNÁNDEZ, E. FORNIÉS MENÉNDEZ*, \\ L. CALLOL SÁNCHEZ
}

Servicios de Neumología. Hospital del Aire y *Hospital de la Princesa. Madrid

\begin{abstract}
OBLITERANS BRONCHIOLITIS WITH IDIOPATHIC ORIGING PNEUMONIA. UTILITY AND PROFITABLENESS OF DIAGNOSTIC TECHNIQUES AND PROCEDURES IN 20 PATIENTS
\end{abstract}

\section{RESUMEN}

Objetivo: Estudio retrospectivo de la utilidad y rentabilidad de las técnicas y procedimientos diagnósticos en pacientes con BONO idiopática.

Material y métodos: Pacientes con diagnóstico histológico de BONO idiopática periodo (1992-1999). Realizándose análisis demográficos, manifestaciones clínicas, laboratorio, funcionales, radiológicas, procedimientos y técnicas de diagnóstico, evolución y mortalidad

Resultados: 20 pacientes (55\%) M, (45\%) V. Edad $64 \pm 15$ años, $50 \%$ fumadores. Intervalo entre inicio clínico y diagnóstico $51 \pm 30$ días. Un cuadro pseudogripal, junto a fiebre, tos, disnea y estertores crepitantes fueron las manifestaciones más frecuentes. Leucocitosis (10.494 \pm 2.642) células $/ \mathrm{mm}^{3}$,VSG $85 \pm 86 \mathrm{UI} / \mathrm{L}, \mathrm{LDH} 309 \pm 86 \mathrm{UI} / \mathrm{L}$. Gasometría arterial: $\mathrm{PaO}_{2} 64 \pm 9, \mathrm{PaCO}_{2} 35 \pm 3 \mathrm{~mm}$ de $\mathrm{Hg}$. Función respiratoria: CVF $81 \pm 25 \%$, VEF $1 / C V F 78 \pm 17 \%$, DLCO $74 \pm 9 \%$, KCO $77 \pm 4 \%$. Rx: opacidades alveolares múltiples bilaterales $(75 \%)$, nodulares solitarias $(15 \%)$ e intersticiales $(10 \%)$. Fibrobroncoscopia y lavado broncoalveolar 10/20 (50\%). Biopsia transbronquial 12/20, siendo diagnóstica en $(60 \%)$. Videotoracoscopia $1 / 20(5 \%)$ y biopsia abierta pulmonar $7 / 20$ $(35 \%)$. Exacerbaciones $8 / 20(40 \%)$, falleciendo $3 / 20(15 \%)$ pacientes.

Conclusiones: La BONO idiopática es una entidad benigna, clínicamente inespecífica asociada a hipoxemia moderada y opacidades alveolares bilaterales. La biopsia transbronquial constituye una técnica diagnóstica alternativa a la biopsia abierta pulmonar en un contexto clínico-radiológico compatible. La respuesta al tratamiento es buena, presentando frecuentes exacerbaciones y baja mortalidad.

PALABRAS CLAVE: Bronquiolitis obliterante con neumonía organizativa. Biopsia abierta pulmón. Biopsia transbronquial. Videotoracoscopia.

\section{ABSTRACT}

Objective: To assess retrospectivily the utility and the profitableness of the diagnostic procedures and techniques in patients with idiopathic $B O O P$.

Material and methods: We studied epidemiologycal features, clinical manifestations, respiratory function tests, chest radiology, and other diagnostic procedures and techniques, as well as the evolution and the mortality rates in patients with histologycal diagnosis of idiopathic BOOP during the period 1992-1999.

Results: 20 patients $(55 \% \mathrm{~F}, 45 \% \mathrm{M})$. Age: $64 \pm 15$ years old, $50 \%$ smokers. The mean period elapsed since the beginning of the symptoms and the time of diagnosis was $51 \pm 30$ days. The clinical presentation is of a subacute influenza-like illness, with fever, cough, dysnea and crackles. The more common abnormalities were. WBC $\left(10,949 \pm 2,6429 \mathrm{~mm}^{3}, \mathrm{ESR}\right.$ $85 \pm 86 \mathrm{Ul} / \mathrm{L}, \mathrm{LDH} 309 \pm 86 \mathrm{UI} / \mathrm{L}$. Arterial bood gas: $\mathrm{PaO}, 64 \pm 9, \mathrm{PaCO}$ $35 \pm 3 \mathrm{~mm} \mathrm{Hg}$. Pulmonary function tests: VCF $81 \pm 25 \%$, FEV V1/VCF 78 $\pm 17 \%$, TLCO $74 \pm 9 \%$, TLCO sb $77 \pm 4 \%$. Radiological evaluation: bilateral multiple patchy alveolar opacities $75 \%)$, solitary focal lesion (15\%) and interstitial opacities (10\%). The bronchoscopy and BAL 10/20 (50\%). Transbronchial biopsy 12/20 wit diagnosis (60\%). The video assisted tho racoscopic lung biopsy 1/20 (5\%) and open lung biopsy $7 / 20$ (35\%). Recurrences $8 / 20(40 \%)$. Mortality rate $3 / 20(15 \%)$.

Conclusions. The idiopathic BOOP is a benign entity clinyc inespe cific, assocIated to hypoxemia and bilateral alveolar opacities. The transbronchial biopsy is a diagnostic procedure alternative to the open lung biopsy, in a compatible clinico-radiological context. The response to treatment is acceptable although it presents frequent exacerbations, and low mortality rate.

KEY WORDS: Bronchiolitis obliterans origing pneumonia. Open lung biopsy. Transbronchial biopsy. Videotoracoscopic

Jareño Esteban J, Zamora García E, Chillón Martín Ma. J, Pérez Amor E, Villegas Fernández F, Forniés Menéndez E, Callol Sánchez L. Bronquiolitis obliterante con neumonía organizativa idiopática. Utilidad y rentabilidad de técnicas y procedimientos diagnósticos en una serie de 20 pacientes. An Med Interna (Madrid) 2001; 18: 63-68.

\section{INTRODUCCIÓN}

La bronquiolitis obliterante con neumonía organizativa focal (BONO), representa para los neumólogos una nueva entidad, cuya descripción inicial se debe a Epler (1), tras los hallazgos clínicos-histológicos observados en una serie publicada en 1985. Desde su descripción muchas han sido las referencias nacionales e internacionales a esta entidad y en ocasiones objeto como en nuestro país de diversos trabajos (2-6).

Trabajo aceptado: 18 de Julio de 2000

Correspondencia: J. Jareño Esteban. C/ Avd. Plaza de Toros, 6-1 B. 28805 Alcalá de Henares (Madrid). 
La BONO define a un proceso inflamatorio inespecífico que afecta a la luz de las pequeñas vías aéreas y espacios aéreos distales, con presencia en su interior de pseudopólipos de tejido conectivo fibromixoide de degranulación $(1,6)$. Generalmente se presenta como formas idiopáticas, aunque en ocasiones se describe asociada a otras entidades. En nuestro país son cada vez más numerosos los casos publicados, aunque escasas las series en donde se describen y comparan sus manifestaciones, procedimientos de diagnóstico y evolución $(7,8)$.

\section{MATERIAL Y MÉTODOS}

Entre el 1 de enero de 1992 y el 31 de diciembre de 1999, fueron diagnosticados 20 pacientes de BONO idiopática, una vez excluidas los casos asociados a otras entidades. En todos ellos se confirmó el diagnóstico histológico, realizándose una revisión de las manifestaciones clínicas, hallazgos de laboratorio y función respiratoria (Master Lab-Jaeger) (espirometría simple y curva flujo volumen, difusión pulmonar y Gasometría arterial). El estudio radiológico, realizado en todos los pacientes, incluyo Rx y TC de tórax (TC-SEE 9000). Los hallazgos radiológicos fueron clasificados: consolidación alveolar, patrón intersticial, patrón mixto y lesión pulmonar solitaria; otras manifestaciones más infrecuentes fueron así mismo constatados. La interpretación de los hallazgos radiológicos fue realizado por dos especialistas en radiología torácica. Un estudio fibrobroncoscópico (FB-Olympus) fue realizado en todos los pacientes, realizándose lavado broncoalveolar (LBA) y biopsia transbronquial (BTB), en los lóbulos y segmentos pulmonares afectados según confirmación previa del TC de tórax. En aquellos pacientes en los cuales no se obtuvo el diagnóstico mediante BTB, se realizó según los casos videotoracoscopia, minitoracotomía diagnóstica o toracotomía con resección de la lesión pulmonar.

Excepto en cuatro casos, todos los pacientes recibieron tratamiento con corticoides (prednisona $1 \mathrm{mg} . \mathrm{kg}^{-1}$ día), reduciéndose progresivamente la dosis según la respuesta obtenida. Ninguno de los pacientes recibió tratamiento con cistostáticos. Excepto en un caso en el cual no se pudo realizar un seguimiento extrahospitalario, el resto de los pacientes fueron controlados en régimen ambulatorio.

\section{RESULTADOS}

\section{CARACTERÍSTICAS GENERALES}

Población: 20 pacientes con BONO idiopática. Edad media $(64 \pm 15)$ años; $11 \mathrm{M}(55 \%)$ y $9 \mathrm{~V}(45 \%)$. Diez pacientes eran fumadores activos $(50 \%)$ y los otros 10 restantes exfumadores o no fumadores (Tabla I).

\section{MANIFESTACIONES CLÍNICAS}

Un periodo de $51 \pm 30$ días transcurrió entre el inicio de las manifestaciones clínicas y el diagnóstico histológico. Los síntomas más frecuentes por orden de frecuencia fueron: inicio pseudogripal (84\%), tos (75\%), fiebre $70 \%$, disnea $(50 \%)$, pérdida de peso $(40 \%)$, dolor torácico $(40 \%)$, sudoración (30\%). En la exploración: estertores $(60 \%)$, disminución del murmullo respiratorio (40\%), roncus y sibilancias (10\%), en ningún paciente se observaron acropaquias (Tabla I).
TABLA I

CARACTERÍSTICAS GENERALES Y M ANIFESTACIO NES CLIINICAS EN UNA SERIE DE 20 PACIENTES CON BO NO IDIOPÁTICA

\begin{tabular}{lr}
\hline Sexo & $55 \% \mathrm{M}, 45 \% \mathrm{~V}$ \\
Edad & $64 \pm 15$ años \\
Fumador/Ex, No fumador & $50 \%-50 \%$ \\
Intervalo inicio clínico diag. & $51 \pm 30$ días \\
Pseudogripal & $80 \%$ \\
Fiebre & $70 \%$ \\
Tos & $75 \%$ \\
Disnea & $50 \%$ \\
Pérdida de peso & $40 \%$ \\
Dolor torácico & $40 \%$ \\
Sudoración & $30 \%$ \\
Estertores & $60 \%$ \\
M urmullo disminuido & $40 \%$ \\
Roncus y sibilancias & $10 \%$ \\
\hline
\end{tabular}

\section{LABORATORIO}

El recuento leucocitario, demostró una leve leucocitosis $\left(10.494 \pm 2.642\right.$ células $\left./ \mathrm{mm}^{3}\right)$, sin eosinofilia en sangre periférica, con incremento de la VSG $\left(85 \pm 37 \mathrm{~mm} \cdot \mathrm{h}^{-1}\right)$. No se objetivaron alteraciones en las enzimas hepáticas y sólo ocasionalmente se observaron incrementos en la LDH (309 \pm 86 UI/L) (Tabla II).

\section{TEST DE FUNCIÓN PULMONAR}

La espirometría simple y curva flujo volumen fue realizado en 19 pacientes (95\%). El 36\% presentaban patrón ventilatorio obstructivo, el $32 \%$ restricción pulmonar, presentando el $32 \%$ restante una espirometría normal. La difusión pulmonar DLCO $(74 \pm 9 \%)$, KCO $(77 \pm 4 \%)$ presentó una disminución en el $44 \%$ de los pacientes, siendo el resto normal. Gasometría arterial reveló: $\mathrm{PaO}_{2}(64 \pm 9) \mathrm{mm}$ de $\mathrm{Hg}, \mathrm{PaCO}_{2}(35 \pm 3) \mathrm{mm}$ de $\mathrm{Hg}$. En cinco de los pacientes (25\%) se objetivó insuficiencia respiratoria, presentando el resto una hipoxemia moderada con normocapnia (Tabla II).

\begin{tabular}{|c|c|}
\hline \multicolumn{2}{|c|}{ TABLA II } \\
\hline \multicolumn{2}{|c|}{$\begin{array}{l}\text { MANIFESTACIONES ANALÍTICAS Y FUNCIONALES EN } \\
\text { PACIENTES CON BONO IDIOPÁTICA }\end{array}$} \\
\hline Laboratorio & \\
\hline $\begin{array}{l}\text { Leucocitos } \\
\text { VSG } \\
\text { LDH } \\
\text { Función pulmonar } \\
\mathrm{PaO}_{2} \\
\mathrm{PaCO}_{2} \\
\text { CVF } \\
\text { FEV1/CVF } \\
\text { DLCO } \\
\text { KCO }\end{array}$ & $\begin{array}{r}10.494 \pm 2.642 \text { células. } \mathrm{mm}^{3} \\
85 \pm 86 \mathrm{UI} / \mathrm{L} \\
309 \pm 86 \mathrm{UI} / \mathrm{L} \\
64 \pm 9 \mathrm{~mm} \text { de Hg} \\
35 \pm 3 \mathrm{~mm} \text { de Hg} \\
81 \pm 25(\%) \\
78 \pm 17(\%) \\
74 \pm 9(\%) \\
77 \pm 4(\%)\end{array}$ \\
\hline
\end{tabular}




\section{MANIFESTACIONES RADIOLÓGICAS}

La Rx y TC de tórax fueron realizados en todos los pacientes. La consolidación alveolar fue el hallazgo más frecuente, presentándose en 15 pacientes $(75 \%)$, opacidad alveolar solitaria en tres $(15 \%)$, patrón intersticial en uno (5\%) y finalmente patrón mixto en otro $(5 \%)$. La afectación radiológica fue predominantemente bilateral $14(70 \%)$ y unilateral en 6 $(30 \%)$. Una distribución periférica y subpleural de los infiltrados se constató en 13 casos $(65 \%)$, con presencia de infiltrados migratorios en $8(40 \%)$. Otros hallazgos más infrecuentes en presentación: cavitación, derrame pleural y adenopatías mediastínicas se observaron en el (15\%) de los pacientes. La afectación pulmonar demostró localización de los infiltrados en: LSD (65\%), LMD (30\%), LID (55\%), LSI (35\%), LII (45\%) (Tabla III) (Figs. 1,2).

\section{TABLA III}

\section{ANIFESTACIO NES RADIO LÓ GICAS (RX-TC) DE TÓ RAX EN PACIENTES CON BO NO IDIOPÁTICA}

O pacidades alveolares múltiples $\quad 75 \% \quad$ O pacidades bilaterales $\quad 70 \%$ 0 pacidades-intersticiales $\quad 10 \% \quad 0$ pacidades unilaterales $\quad 30 \%$ Lesión nodular solitaria $\quad 15 \% \quad$ Infiltrados migratorios $\quad 40 \%$ Cavitación $\quad 15 \%$ Distribución periférica $65 \%$ Adenopatías-mediastínicas $\quad 15 \%$ Derrame pleural $\quad 15 \%$

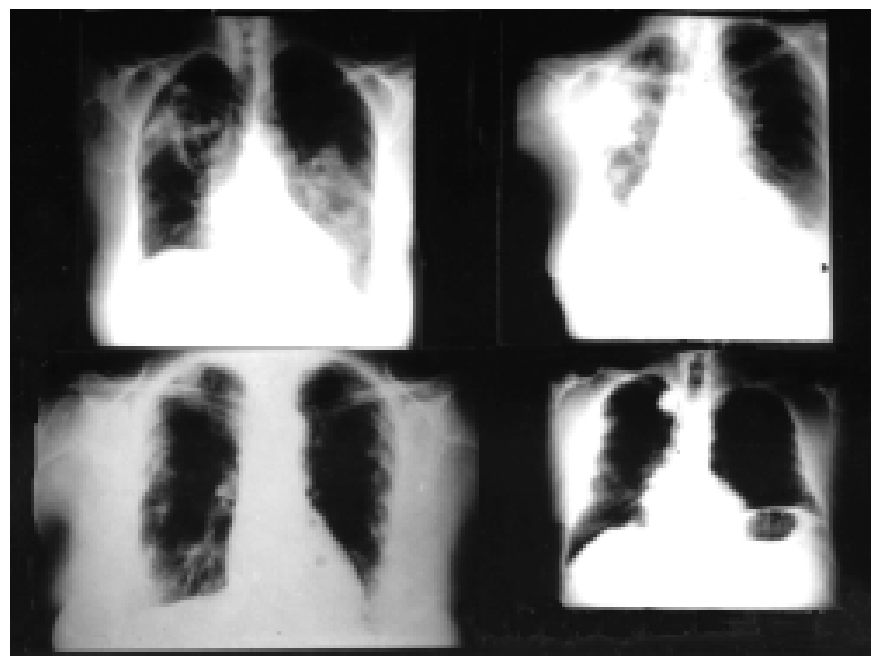

Fig. 1. Rx de tórax con presencia de: a) opacidades alveolares; b) unilaterales; c) opacidades intersticiales; d) opacidad alveolar solitaria.

\section{PROCEDIMIENTOS DIAGNÓSTICOS}

Un estudio $\mathrm{FB}$ fue practicado a todos los pacientes y LBA en $10(52 \%)$. El recuento celular diferencial obtenido reveló: macrófagos $(70 \pm 18 \%)$, neutrófilos $(8 \pm 8 \%)$, linfocitos $(17 \pm 12 \%)$ y eosinófilos $(5 \pm 5 \%)$ (Tabla IV).

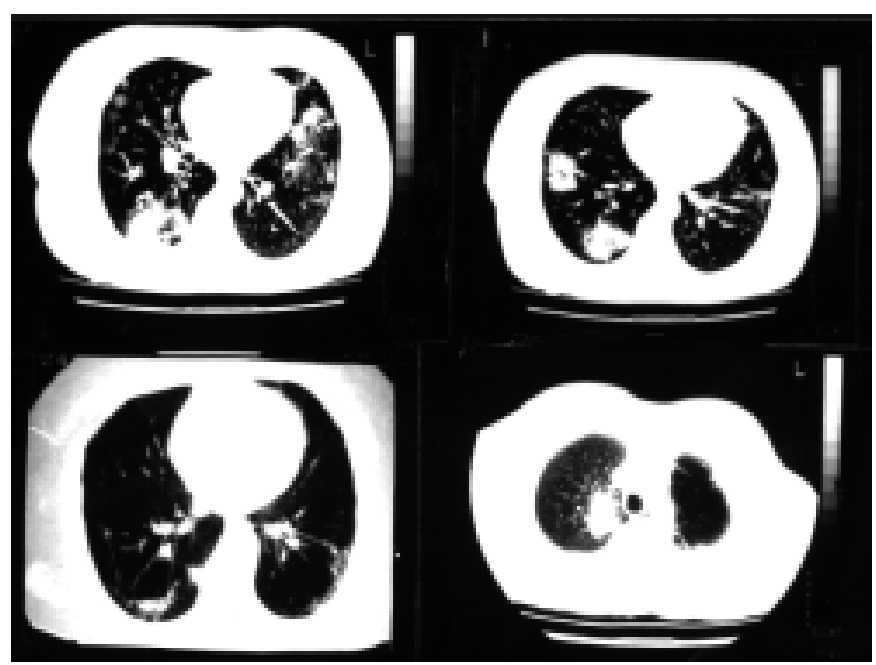

Fig. 2. TC torácico con manifestaciones de BONO: a) opacidades alveolares bilaterales: b) unilaterales: c) opacidades intersticiales y d) opacidad solitaria.

\begin{tabular}{|c|c|}
\hline \multicolumn{2}{|l|}{ TABLA IV } \\
\hline \multicolumn{2}{|c|}{$\begin{array}{l}\text { TÉCNICAS Y PRO CEDIM IENTOS DIAGNÓ STICO S EN } \\
\text { PACIENTES CON BONO IDIO PÁTICA }\end{array}$} \\
\hline FB-Lavadobroncoalveolar (LBA) & \\
\hline $\begin{array}{l}\text { M acrófagos } \\
\text { Neutrófilos } \\
\text { Linfocitos } \\
\text { Eosinófilos } \\
\text { FB-Biopsia transbronquial } \\
\text { Videotoracoscopia } \\
\text { Toracotomía y biopsia pulmonar abierta }\end{array}$ & $\begin{array}{r}69 \pm 18(\%) \\
8 \pm 8(\%) \\
19 \pm 12(\%) \\
5 \pm 5(\%) \\
12 / 20(60 \%) \\
1 / 20(5 \%) \\
7 / 20(35 \%)\end{array}$ \\
\hline
\end{tabular}

La FB con BTB se practicó en los 20 pacientes, realizándose en las áreas con mayor afectación radiológica según información proporcionada por el TC torácico. Fue positiva, confirmando la existencia de lesiones compatibles con BONO en 12 pacientes (60\%). En los 8 pacientes restantes con BTB negativa, se realizó: videotoracoscopia en 1 caso (5\%), minitoracotomía pulmonar diagnóstica en 4 (20\%) y en los 3 pacientes restantes se realizó toracotomía con extirpación pulmonar de la lesión solitaria pulmonar (15\%).

En seis pacientes los hallazgos histológicos de BONO se asociaron con la presencia de: neumonía intersticial usual en un caso (NIU) (5\%) y en los cinco restantes con neumonía intersticial no específica (NSIP) (25\%).

\section{TRATAMIENTO Y EVOLUCIÓN}

Todos los pacientes excepto en cuatro casos recibieron tratamiento con corticoides (prednisona $1 \mathrm{mg} \cdot \mathrm{kg}^{-1}$ día). Las excepciones correspondieron: en un caso por resolución espontánea y en otros tres por corresponder a formas de BONO con presentación pulmonar solitaria, requiriendo para su diagnostico toracotomía diagnóstica con resección pulmonar. 
El control ambulatorio se realizó en todos los pacientes, excepto en un caso, con un seguimiento de (30-2190) X 660 días. Ocho pacientes $(40 \%)$ presentaron buena evolución, una vez finalizado el tratamiento, no observándose recidivas tras el mismo. En total se constataron 18 reactivaciones de BONO en 8 pacientes $(40 \%)$. En ninguno de los casos de BONO solitaria, se apreciaron recidivas en su evolución.

Al finalizar el seguimiento cinco pacientes continuaban en tratamiento con corticoides, en dos casos con pauta descendente y en los tres restantes con mantenimiento (10-20 mg prednisona/día). Tres pacientes fallecieron en el seguimiento (15\%), no atribuible a BONO (Tabla V).

\begin{tabular}{lcc}
\multicolumn{3}{c}{ TABLA V } \\
\multicolumn{3}{c}{$\begin{array}{c}\text { EVO LUCIÓ N Y M O RTALIDAD EN PACIENTES } \\
\text { CON BO NO IDIO PÁTICA }\end{array}$} \\
\hline No & $\%$ \\
\hline $\begin{array}{l}\text { M ortalidad } \\
\text { Recidivas }\end{array}$ & $3 / 20$ & $(15 \%)$ \\
\hline
\end{tabular}

\section{DISCUSIÓN}

La BONO constituye un síndrome infrecuente, de inicio subagudo, asociado generalmente a infiltrados pulmonares bilaterales, distribuidos periféricamente y con tendencia a la migración. Un defecto ventilatorio restrictivo, puede asociarse a obstrucción variable, con presencia histológica de un tejido de granulación en los ductus alveolares y presencia distal de áreas con neumonía organizada.

La serie que presentamos resume las características clínicas de 20 pacientes con BONO idiopática, presentando un ligero predominio en mujeres $(55 \%)$, con una media de edad de 64 años y elevado porcentaje de pacientes fumadores $(50 \%)$.

Cuando comparamos los resultados obtenidos con series más amplias Epler (1), Alasaly (9), Yamamoto (10), Costabel (11) observamos como hombres y mujeres son igualmente afectados, desconociendo por el momento los factores predisponentes; presentando nuestra serie una edad media algo superior respecto a otros autores (50-60 años). El tiempo transcurrido entre el inicio clínico y el diagnóstico situado en una media de 51 días, se sitúa en el rango habitual referido por Epler para esta entidad (2-10 semanas) (1). Las manifestaciones clínicas y de exploración son muy similares en todas las series publicadas; con pequeñas diferencias entre ellas $(1,9,10)$. La hemoptisis no observada en nuestra serie, es un hallazgo infrecuente observándose en formas de BONO con lesión solitaria $(11,12,15)$.

Los hallazgos de laboratorio son en general inespecíficos, mostrando una discreta leucocitosis, sin eosinofilia, acompañado de alteraciones en la VSG, LDH, etc. Algunos autores han referido alteraciones en el funcionalismo hepático, en posible relación con factores ambientales, situación que no hemos observado en nuestra serie (14). La función pulmonar viene caracterizada por un defecto ventilatorio restrictivo, con disminución de la difusión; un grado variable de obstrucción puede ser observado. Nuestros resultados confirmaron una restricción pulmonar en un $32 \%$, con disminución en la difusión pulmonar en el $44 \%$ de los pacientes. Posiblemente los resultados obtenidos puedan estar influenciados por el alto porcentaje de población fumadora, que supera sensiblemente al de otras series publicadas. Una hipoxemia de intensidad moderada (79\%), con presencia de normocapnia fue predominante, presentando cinco pacientes $(25 \%)$, criterios de insuficiencia respiratoria. La hipoxemia severa en la BONO puede reflejar una grave enfermedad pulmonar o ser una manifestación en casos con lesiones localizadas $(1,6,9-11,15)$.

Característicamente la BONO se ha asociado con una amplia variedad de manifestaciones radiológicas (15). Las opacidades alveolares son usualmente múltiples, asimétricas, parcheadas, distribuyendose subpleural y periféricamente, pudiendo presentarse como consolidación o imagen en vidrio deslustrado,con broncograma aéreo, son las más frecuentes en presentación, mostrando frecuente tendencia a la migración de forma espontánea. Las formas de BONO con presentación unilateral son más infrecuentes (4\%), presentándose en nuestra serie en un $(30 \%)$, porcentaje superior al referido por otros autores $(6,9)$. Un patrón intersticial con opacidades (nodulares, reticulares, mixto, etc.) fue observado solamente en el $(10 \%)$ de los casos; porcentaje sensiblemente inferior a otras series (18-42\%). Finalmente la BONO puede manifestarse como opacidades únicas y solitarias, constituyendo la segunda forma de presentación entre nuestros pacientes. Otros hallazgos radiológicos más infrecuentes pueden ser observados: hiperinsuflación, adenopatías mediastínicas, derrame pleural, cavitación, neumotórax, etc (1,6,9-11,16-19).

A diferencia de la NEC que muestra predisposición por localizarse sus manifestaciones en los lóbulos superiores, en la BONO han sido referidos hallazgos contradictorios. Algunos autores han demostrado una mayor frecuencia de los infiltrados de BONO en los lóbulos inferiores, presentándose las opacidades nodulares solitarias con mayor frecuencia en lóbulos superiores $(11,15)$. Nuestra experiencia confirma estos hallazgos, para las formas solitarias de BONO, siendo los lóbulos inferiores más afectados en las presentaciones bilaterales.

La FB constituye en general, la primera técnica de aproximación en el diagnóstico de BONO; el LBA muestra un incremento en la celularidad, con predominio linfocitario, pudiendo observarse macrófagos espumosos y células plasmáticas. El fenotipo linfocitario muestra disminución del cociente CD4/CD8, con población normal de linfocitos CD57 (+). En general el LBA muestra una baja especificidad (57\%) y sensibilidad $(63 \%)$ en esta entidad, pero su realización constituye una ayuda complementaria inestimable, permitiéndonos su diferenciación con otros procesos (NEC, NH, infecciones etc). Los recuentos celulares obtenidos en nuestros casos demostró un incremento en todas las poblaciones celulares, con moderada linfocitosis, careciendo de experiencia en el estudio de las poblaciones linfocitarias, cuya determinación resulta de gran utilidad en la diferenciación con otras entidades (20-24).

El diagnóstico de la BONO precisa en todos los casos de una confirmación histológica, obtenida por: BTB, videotoracoscopia o preferentemente por minitoracotomía pulmonar. La FB y BTB ampliamente utilizada en el diagnóstico de las enfermedades pulmonares intersticiales difusas (sarcoidosis, neumonitis por hipersensibilidad, histiocitosis, etc.) ha sido cuestionada como procedimiento diagnóstico en la BONO. 
Entre sus principales objeciones destacan, la naturaleza parcheada de la enfermedad, unido al pequeño tamaño en las muestras obtenidas dificultaría en gran medida su diagnóstico. No obstante son cada vez más frecuentes y numerosos los casos publicados cuyo diagnóstico fue obtenido mediante BTB. Su realización precisa de un control radiológico de las zonas afectadas, confirmadas previamente por Rx-TC antes de la biopsia pulmonar, dado la tendencia migratoria de los infiltrados en esta entidad e incluso la resolución espontánea. Una sensibilidad del $64 \%$ y una especificidad del $86 \%$ han sido establecidas para esta técnica (24) y su rentabilidad variable dependiendo de las series nacionales o internacionales publicadas, oscilando entre un (29-90\%) (1,7-12,20-33,34). En nuestra serie su rentabilidad se situó en un $60 \%$, considerando los hallazgos histológicos como diagnósticos, si el contexto clínico radiológico era altamente sugestivo de BONO. El diagnóstico histológico mediante BTB podría ser asumido, siempre que las muestras obtenidas contenga bronquiolos respiratorios, conductos alveolares y alveolos, precisando de hallazgos característicos de esta entidad (22).

La biopsia pulmonar obtenida mediante videotoracoscopia o minitoracotomía debería ser reservada para los casos con diagnostico FB y BTB negativa.

La videotoracoscopia como técnica diagnóstica cada vez más empleada en el diagnóstico de las enfermedades pulmonares ha sido poco utilizada en la BONO, siendo escasos los diagnósticos obtenidos por este procedimiento; beneficiándose en nuestra serie, un solo paciente. Constituyendo la BONO una entidad con frecuente afectación pulmonar periférica, bien podría la videotoracoscopia constituirse en el futuro como uno de los procedimientos diagnósticos de referencia, incluso desplazando y reservando la minitoracotomía para los casos con insuficiencia respiratoria o sometidos a ventilación mecánica. Los pacientes podrían beneficiarse de menores complicaciones y de un corto y más cómodo postoperatorio, todo ello redundando en menor tiempo de hospitalización y coste económico (36,38-41).

Finalmente la biopsia pulmonar abierta mediante minitoracotomía, constituye la técnica y procedimiento más estandarizado y de elección en la BONO. Su realización varía con las series publicadas (67-100\%), situándose para nuestra serie en un $35 \%(1,9-11,20)$

El diagnóstico de BONO sin confirmación histológica está rara vez justificado, podría ser considerado ante situaciones complejas en pacientes ancianos o en situación crítica.
Un apartado especial merece la BONO como forma de presentación solitaria. Existe unanimidad, en la necesidad de un abordaje más invasivo de estos casos, mediante la realización de biopsia abierta y exéresis quirúrgica, al plantearse su diagnóstico diferencial fundamentalmente con el carcinoma broncogénico entre otras entidades, situación que se presentó en tres de nuestros casos $(33,37,38)$.

El pronóstico y evolución de la BONO idiopática es bueno, con baja mortalidad (3-15\%) $(1,37)$, comparado con formas secundarias o asociadas. Resoluciones espontáneas han sido observadas en pacientes en tratamiento prolongado con eritromicina. En general responden al tratamiento con corticoides un 60-70\% de los casos con respuestas parciales en un $30 \%$. Formas más agresivas lo constituyen casos no idiopáticos y asociados a conectivopatías, fármacos, etc., con presencia de insuficiencia respiratoria, afectación intersticial, mínima linfocitosis en el LBA y asociación a NIU e intensa remodelación del parénquima pulmonar $(3,36,37,39-41)$. Con frecuencia podemos observar casos de BONO asociado a formas de (NSIP) (42\%), en general estos casos presentarían un buen pronóstico y buena respuesta al tratamiento (46-48). Las recurrencias pueden presentarse a lo largo de la evolución, pudiendo ser observadas hasta pasados varios años de establecido el diagnóstico. La reducción o supresión precoz del tratamiento con corticoides, asociada a cambios histológicos de NIU-NSIP constituyeron en nuestra experiencia una de las causas más frecuentes de recurrencias. La evolución hacia la fibrosis pulmonar puede observarse en un (20-25\%) de lo casos. Ninguno de nuestros pacientes ha presentado por el momento esta evolución, excepto el paciente que debutó con BONO asociada a NIU, precisando estos casos de un prolongado tratamiento de mantenimiento con corticoides a dosis bajas.

Podemos concluir que la BONO idiopática, se comporta en nuestra experiencia como una enfermedad inflamatoria inespecífica, de curso generalmente benigno. El LBA aún careciendo de utilidad diagnóstica nos permite su diferenciación con otras entidades (NEC, $\mathrm{NH}$, etc.).

La BTB y especialmente la videotoracoscopia pueden constituir en el futuro como procedimientos diagnósticos alternativos a la biopsia pulmonar abierta, utilizada actualmente como diagnóstico de referencia. Las recurrencias en su evolución, obligan a un tratamiento y seguimiento prolongado para los casos con curso más desfavorable.

\section{Bibliografía}

1. Epler GR, Colby TV, Mc Loud T, Carrington C, Gaensler E. Bronchiolitis obliterans organizing pneumonia. N Engl J Med 1985; 312: 152-158.

2. Izumi T. Proceedings of the International Congress on Bronchiolitis Obliterans Organizing Pneumonia. Chest 1992; 102: 1-50.

3. Candeira S, Hérnandez L, Gil J. Bronquiolitis obliterante. Actualizaciones SEPAR 1996; 2: 27-49.

4. Orriols R, Bravo C. Bronquiolitis obliterante: dificultades de la definición. Arch Bronconeumol 1995; 31: 1-2.

5. Álvarez Sala JL, Martínez Cruz JMF, Sánchez R, Álvarez Sala R. Bronquiolitis oblite-rante y bronquiolitis obliterante con neumonía organizada. An Med Interna (Madrid) 1998; 15: 224-225.

6. Cordier JF. Organising pneumonia.Thorax 2000; 55: 318-328.
7. Romero V, Alemany LM, Martínez A, Andujar J, Aranda I, Romero S Bronquioitis obliterante: análisis de 18 casos. Arch Bronconeumol 1995; 31: 77.

8. Jareño J, Villegas F, Aspa J, Chillón MJ, Jiménez C, Zuil M, Ancoechea J, Gómez de Terreros FJ, Forniés E, Callol L. Bronquiolitis obliterante con neumonía organizativa focal idiopática. Características clínicas diagnósticas y evolutivas en una serie de 17 casos. Neumomadrid Par 1999; 2: 203-212.

9. Alasaly K, Muller N, Ostrow DN, Champion P, Fitzgerald JM. Cryptogenic Organizing Pneumonia. A report of 25 cases and a rewiew of the literature. Medicine 1995; 74: 201-211.

10. Yamamoto M, Ina Y, Kitaichi M, Harasawa M, Tamura M. Clinical fea tures of Boop in Japan. Chest 1992; 102: 21-25. 
11. Costabel U, Teschler H, Schoenfeld B, Hartung W, Nusch A, Guzman J, et al. Boop in Europe Chest 1992; 102: 14-20.

12. Haro M, Núñez A, Vizcaya M. Hemoptisis y bronquiolitis obliterante con neumonía organizativa. Arch Bronconeumol 1998; 34: 410.

13. Mroz BJ, Sexauer WP, Meade A, Balsara G. Hemoptysis as the presenting symptom in Bronchiolitis Oblietrans Organizing Pneumonia. Chest 1997; 111: 1775-1778.

14. Spiteri MA, Klenerman P, Sheppard MN, Pdaley S, Clarck TJK, Newman-Taylor A. Seasonal cryptogenic organizing pneumonia with biochemical cholestasis. A new clinical entity. Lancet 1992; 340: 281-284.

15. Cordier JF, Loire R, Brune J. Idiopathic bronchiolitis obliterans organizing pneumonia. Definition of characteristic clinical profiles in a series of 16 patients. Chest 1989; 96: 999-1004.

16. Casas L, Álvarez A, Torrens C, Puy R, Sánchez C. Manifestaciones radiológicas de la bronquiolitis obliterante. Rev Clin Esp 1991; 188: 446-449.

17. Lee KS, Kullnig P, Hartman TE, et al. COP. CT findings in 43 patients. AJR. Am J Roentgenol 1994; 162: 543-546.

18. Kofteridis DP, Bouros DE, Vamvakas LN, Stefanaki KS, Voludaki KS, Barbounakis EM, et al. Respiration 1999; 1,5: 379-381.

19. AD Tasker, C DR Flower. Imaging the airways. Hemoptysis, bronchiectasias and small airways disease. Clin Chest Med 1999; 4: 761-773.

20. Costabel U, Teschler H, Guzman J. Bronchioitis obliterans organizing pneumonia (BOOP): the cytological and immunocytological profile of bronchoalveolar lavage. Eur Respir J 1992; 5: 791-797.

21. Nagai S, Aung H, Tanaka S, Satake N, Mio T, Kawatani A, Kusume K, Kitaichi M, Izumi T. Bronchoalveolar lavage cell findings in patients with BOOP and release diseases. Chest 1992; 102S: 32-37.

22. Perez JL, Ancochea J. El lavado broncoalveolar en la enfermedad pulmonar alveolointersticial difusa no infecciosa. Actualizaciones SEPAR 1995; 1: 187-236.

23. Pesci A, Mojori M, Piccoli ML, Casalini A, Curti A, Franchini D, et al. Mast cell in bronchiolitis obliterans organizing pneumonia. Mast cell hyperplasia and evidence for extracellular release of tryptase. Chest 1996; 110: 383-391.

24. Poletti V, Cazzato S, Minicuci N, Zompatori M, Burzi M, Schiattone ML. The diagnos-tic value of bronchoalveolar lavage and transbronquial lung biopsy in cryptogenic organizing pneumonia. Eur Respir J 1996; 9: 2513-2517.

25. Azzam ZS, Bentur L, Rubin A, Ben Izhak O, Alroy G. Bronchiolitis obliterans organizing pneumonia. Diagnosis by transbronchial biopsy. Chest 1993; 104: 1899-1901.

26. Curley FJ, Johal JS, Burke ME, Fraire AE. Transbronchial lung biopsy. Can specimen quality be predicted at the time of biopsy? Chest 1988; 113: $1037-1041$.

27. Antón E, Alkiza R, Altuna E, Marti J. Bronquiolitis obliterante con neumonía en organización idiopática. Experiencia de un hospital comarcal. An Med Interna (Madrid) 1998; 15: 152-154.

28. Olivera MJ, Caballero P, González S, Jareño J, Gómez J, Casanova R. Bronquiolitis obliterante con neumonía organizada. Radiología 1993; 35: 277-298.

29. Izquierdo M, Coloma R, Haro H. Patrón miliar secundario a bronquiolitis obliterante con neumonía organizada. Arch Bronconeumol 1999; 35: 55.

30. Gispert FX, Prtyz MA, Camacho P, Rovira A, Albasanz JA, Riz MJ. Bronquiolitis obliterante con neumonía en organización.Estudio clínico patológico de seis casos. Med Clin 1995; 74: 201-211.
31. Marchante I, Mayoral L, Benítez JA, Lozano F. Bronquiolitis obliterante con neumonía en organización idiopática. An Med Interna (Madrid) 1994; 11: 564-565.

32. Barba MA, Arévalo M, Aguilar X, González G, Vizcaya M, Puras A Bronquiolitis obliterante con neumonía en organización idiopática. Experiencia en un hospital general. Arch Bronconeumol 1995; 31: 481484.

33. Domingo JA, Pérez Calvo J, Carretero JA, Ferrando J, Cay A, Civeira F. Bronchiolitis obliterans organizing pneumonia. An unusual cause of solitary pulmonary nodule. Chest 1993; 103: 1621-1623.

34. Alegre-Martín J, Fernández de Sevilla T, García F, Falco V, Martínez Vázquez JM. Three cases of idiopatic bronchiolitis obliterans with organizing pneumonia. Eur Respir J 1991; 4: 902-904.

35. Bensard DD, McIntyre RC, Waring BJ, Simon JS. Comparison of video thoracoscopic lung biopsy to open lung biopsy in the diagnosis of interstitial lung disease. Chest 1993; 103: 765-770.

36. Carnochan FM, Walker WS, Cameron EWJ. Efficacy of video assisted thoracoscopiclung biopsy: an historical comparison with open lung biopsy. Thorax 1994; 49: 361-363.

37. Krasna MJ, White ChS, Aisner SC, et al. The role of thoracoscopy in the diagnosis of interstitial lung disease. Ann Thorac Surg 1995; 59: 348-351.

38. Callejas MA, Belda J, Baldó X, Canalis E, Catalán M, Gimferrer JM. Biopsia pulmonar en la enfermedad interstiticial difusa:videotoracoscopia frente a toracotomía. Arch Bronconeumol 1996; 32: 10-13.

39. Martín T, Larraga R, Badorrey I, De Andrés JL, Sánchez F, Bello S, Hernández A. La videotoracoscopia frente a la toracotomía en el diagnóstico de la enfermedad pulmonar intersticial difusa. Arch Bronconeumol 1997; 33: 341-345.

40. Cordier JF. Cryptogenic Organizing Pneumonitis. Clin Chest Med 1993; 14: 677-692.

41. Matsubara O, Tan Liu NS, Kenney R, et al. Inflamatory pseudotumors of the lung: progresion from organizing pneumonia to fibrous histyocitoma or to plasma cell granuloma en 32 cases. Hum Pathol 1988; 19: 807-814.

42. King TE, Mortenson RL. Cryptogenic organizing pneumonia. The North American Experience. Chest 1992; 102: 8-13.

43. Cohen AJ, King TE, Downey GP. Rapidly progresive bronchiolitis obliterans with organizing pneumonia. Am J Respir Crit Care Med 1994; 149:1670-1675.

44. Watanabe K, Senju S, Wen FQ, Shiracusa T, Maeda F, Yoshida M. Factors related to the relapse of bronchiolitis obliterans organizing pneumonia. Chest 1998; 114: 1599-1606.

45. Yousem SA, Lohr RH, Colby T. Idiopathic pulmonary fibrosis. Idiopathic bronchiolitis obliterans organizing pneumonia cryptogenic with unfavorable outcome: pathologic predictors. Mod Pathol 1997; 10: 864-871.

46. Katzenstein A LA, Myers JL. Idiopathic pulmonary fibrosis. Clinical relevance of pa-thologic classification. State of the art. Am J Respir Crit Care Med 1998; 157: 1301-1315.

47. Cottin V, Donsbeck AV, Revel D, Loir R, Cordier JL. Non specific Interstitial Pneunia. Individualization of a clinicopathologic entity in a series of 12 patients. AM J Respir Crit Care Med 1998; 158: 1286-1293.

48. Katzenstein A LA, Myers JL. Nonspecific interstitial Pneumonia and the other idiopathic interstitial pneumonias: Clasification and Diagnostic Criteria. Am J Surg Pathol 2000; 24: 1-3. 